Kłosińska Martyna, Kaczyńska Agnieszka. Polycystic ovarian syndrome - management and treatment. Journal of Education, Health and Sport. 2021;11(9):123-130. eISSN 2391-8306. DOI http://dx.doi.org/10.12775/JEHS.2021.11.09.017

https://apcz.umk.pl/czasopisma/index.php/JEHS/article/view/JEHS.2021.11.09.017

https://zenodo.org/record/5484900

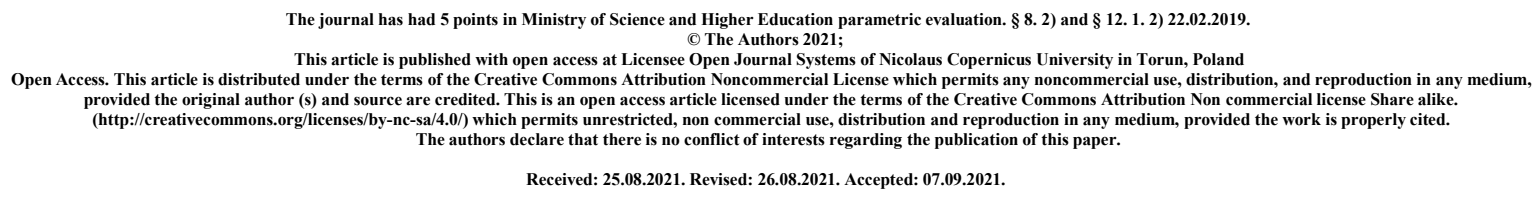

\title{
POLYCYSTIC OVARIAN SYNDROME - MANAGEMENT AND TREATMENT
}

\section{Martyna Kłosińska ${ }^{1}$, Agnieszka Kaczyńska ${ }^{1}$}

Martyna Kłosińska, martynaklosinska69@gmail.com, https://orcid.org/0000-0001-7649$\underline{085 X}$

Agnieszka Kaczyńska, agaagnieszkakk@gmail.com, https://orcid.org/0000-0002-8943-9401

(1) Faculty of Medicine, Medical University of Lublin, Aleje Racławickie 1, 20-059 Lublin, Poland

\section{SUMMARY}

The polycystic ovarian syndrome is an endocrine disorder with a high prevalence affecting reproductive-aged women and adolescent girls. The most common symptoms are infertility, numerous ovarian cysts, hyperandrogenism, menstrual cycle abnormalities. The disease has a great impact on patients, impairing their quality of life. Bearing that in mind, innovative therapeutic approaches are needed. We aim to describe current management and treatment concepts adopted in PCOS therapy. The analysis of previously published studies was conducted by using the PubMed, Google Scholar, and Scopus databases.

Mentioned studies show that lifestyle changes may benefit PCOS therapy in multiple ways. Dietary and exercise interventions have a notable influence on women's body composition as well as biochemical parameters. The reduction of body weight or BMI is a therapeutic achievement likewise patients' contribution to managing PCOS. Pharmacotherapy provides plentiful treatment choices to adopt. Studies show that hormonal contraceptive has a significant impact on both hyperandrogenism and menstrual abnormalities. Metformin is 
proven to reduce BMI, improve LDL levels, and lower the risk of OHSS in women undergoing IVF/ICSI-ET. A meta-analysis proved metformin's monotherapy superiority over clomifene citrate or their combination in treating infertility. However, both substances are not recommended as the first-line choice. Regrettably, many studies mention short of evidence, lack of larger sample sizes, or insufficient duration preventing further research.

The conclusion we draw is that approaches of managing and treating PCOS are constantly advancing and updating. However, there is a necessity for future studies to expand their research by gathering more data and considering diverse cases.

Key words: polycystic ovarian syndrome; endocrine productive disorder; infertility; hyperandrogenism; hormonal contraceptive; metformin; clomifene citrate

\section{INTRODUCTION AND PURPOSE}

Polycystic ovarian syndrome (PCOS) is an endocrine-metabolic pathology that has an increasing prevalence affecting between $8 \%$ and $13 \%$ of reproductive-aged women and 6$18 \%$ of adolescent girls.[1] To diagnose the disorder at least two of the three following criteria must be present: chronic oligo-/anovulation, hyperandrogenism (clinical or biological), and polycystic ovaries.[2] However, the occurrence of polycystic ovaries is a common ailment that does not accurately indicates the presence of the PCOS condition, as it is neither needed nor sufficient in order to make the diagnosis. Moreover, the name of the disorder does not imply any of the significant metabolic manifestations.[3, 4]

There are four recognised phenotypes of PCOS: full-blown syndrome, phenotype A hyperandrogenism (HA) + oligo-/anovulation (OA) + polycystic ovaries at ultrasound (PCO); phenotype B - HA + OA; phenotype C - OA + PCO; phenotype D - HA + PCO.[5]

The most common symptoms of PCOS are acne, alopecia, anxiety, depression, hirsutism, hyperinsulinemia, infertility, menstrual irregularities, obesity, oligomenorrhoea.[69] Multiple diseases' symptoms may mimic features of PCOS, for instance, thyroid disease, hyperprolactinemia, or congenital adrenal hyperplasia. Therefore, the diagnosis of PCOS must be made of exclusion. The disease is frequently underdiagnosed which might lead to the aggravation of symptoms as well as the decrease in the quality of patients' life.[10] A fast and proper diagnosis decreases the risk of future manifestation of associated metabolic and cardiovascular risks which are direct consequences of untreated PCOS. In addition, it enables 
an appropriate medical intervention with a strict relation to the woman's age, reproductive status, and her concerns.[11]

In this review, we aim to present the current practice of management and treatment of PCOS focusing on various ways the disease is being approached. The analysis and presentation of the previously published studies were conducted by browsing through PubMed, Google Scholar, and Scopus databases.

\section{DESCRIPTION OF THE STATE OF KNOWLEDGE}

\subsection{LIFESTYLE CHANGES}

The first and the simplest method of managing PCOS is completely dependent on the patient. This disease and its' repercussions demand implementing various changes in one's lifestyle in order to fully benefit from medicaments. Modifications consist of an appropriate and healthy diet, regular exercises, weight loss in case of obesity, behavioural interventions for example quitting smoking and drinking alcohol.

In 2019 Lim et al. presented a meta-analysis whose aim was to observe the effectiveness of overall lifestyle changes in the progress of management of PCOS. The factors considered were reproductive, metabolic, anthropometric as well as the quality of life. 15 studies with 498 participants were included. 10 studies were concerned about the impact of physical activity as oppose to minimal or zero dietary and behavioural intervention. 5 studies assessed a combination of diet, exercises, and behavioural interventions in comparison to minimal intervention. It was conducted that lifestyle intervention may enhance a secondary reproductive factor - the free androgen index (FAI) (MD -1.11, 95\% (CI) -1.96 to -0.26). Moreover, it can contribute to reducing weight (MD $-1.68 \mathrm{~kg}, 95 \% \mathrm{CI}-2.66$ to -0.70 ) and body mass index (BMI) $(-0.34 \mathrm{~kg} / \mathrm{m} 2,95 \% \mathrm{CI}-0.68$ to -0.01$)$. The lifestyle change's influence on glucose tolerance is uncertain (SMD -0.02, 95\% CI -0.38 to 0.33).[12] The outcome of this meta-analysis is consistent with the literature.

In 2018 Benham et al. conducted a meta-analysis assessing exercise training's effect on reproductive and cardiometabolic parameters in women with PCOS. They included 517 screened abstracts and 14 studies including 617 patients. The results showed the improvement in lipid profiles as well as decrease in waist circumference, systolic blood pressure, and fasting insulin. Due to insufficient data, only a semi-quantitive analysis of reproductive outcomes was conducted and it presented a possible improvement in menstrual regularity, pregnancy, and ovulation rates. However, the authors claim it remains unclear.[13] 
In 2020 Dos Santos et al. presented a meta-analysis to examine different types of training's impact on women's body composition and reproductive function. There were 10 studies with 533 participants included with various types of exercises. It resulted in low certainty evidence for little to no effect on reproductive factors and moderate certainty evidence of BMI reduction (BMI SMD $-0.35,95 \%$, CI -0.56 to $-0.14, \mathrm{P}=.001$ ). The authors expressed the necessity for future studies with longer duration and larger sample sizes.[14]

In 2020 Shang et al. conducted a meta-analysis in order to evaluate a possible diet intervention's effect on reducing insulin resistance (IR) in women with PCOS. 19 studies with 1193 patients were included. The outcome showed a significant correlation between diet and improvements in IR and body composition including BMI. They observed an association between the length of duration of treatment and improvement of parameters. The conclusion was a suggestion of the benefits and effectiveness of professional dietary advice to all PCOS patients.[15]

Nowadays women's contribution to the management of PCOS is a great asset to their therapy. Mentioned studies showed that through diet and exercise interventions patients have a direct impact on their body composition and improvement of biochemical parameters. For that reason, new medical approaches and innovative methods can be established.

\subsection{PHARMACOTHERAPY}

The main concerns of the PCOS treatment are infertility and menstrual abnormalities in addition to hyperandrogenism resulting in hirsutism, acne, and being prone to weight gain. Therefore, the pharmacotherapy of this disease is a number of various medicaments individually and appropriately combined for every patient. The first-line and most commonly used therapeutic options are hormonal contraceptives, clomiphene, and metformin.[16]

In 2016 Khalifah et al. presented a meta-analysis whose aim was to assess and evaluate the use of metformin versus oral contraceptive pills (OCPs) in the therapy of PCOS in adolescents aged 11 to 19 years. There were 4 studies with 170 patients included. The treatment with OCPs resulted in modest improvement of menstrual cycle frequency (WMD= $0.27, \mathrm{P}<.01,95 \%, \mathrm{CI}-0.33$ to -0.21$)$ and mild reduction of acne scores (WMD $=0.3, \mathrm{P}=.02$, $95 \%$ CI 0.05 to 0.55$)$. The therapy with metformin showed a greater BMI reduction (WMD = $0.3, \mathrm{P}=.02,95 \% \mathrm{CI} 0.05$ to 0.55$)$ which was correlated to decreased dysglycemia prevalence (RR: $0.41, \mathrm{P}=.02,95 \% \mathrm{CI} 0.19$ to 0.86 ) and improvement of total cholesterol and lowdensity lipoprotein levels. The two medicaments occurred to have a similar impact on 
hirsutism. It was stated in conclusion that current data is of either low or very low quality, therefore the treatment choice ought to be individually and carefully implemented.[17]

In $2020 \mathrm{Wu}$ et al. conducted a meta-analysis examining metformin's association with outcomes of in vitro fertilisation or intracytoplasmic sperm injection and embryo transfer (IVF/ICSI-ET), which are preferred methods of infertility treatment in case of failure of pharmacotherapy. 12 studies including 1123 participants were included. The analysis presented a lower risk of ovarian hyperstimulation syndrome (OHSS) in women randomised to metformin (OR, 0.43; 95\% CI, 0.24-0.78), although this was not significant for patients with BMI of less than 26 (OR, 0.67; 95\% CI, 0.30-1.51). The observation of both clinical pregnancy rate $(\mathrm{OR}, 1.24 ; 95 \% \mathrm{CI}, 0.82-1.86)$ and live birth rate $(\mathrm{OR}, 1.23$; 95\% CI, 0.742.04) showed no statistically significant difference. In overall, metformin stands as a medical option for women with PCOS undergoing IVF/ICSI-ET and may be more preferable for patients with BMI greater than 26. [18]

In 2009 Palomba et al. presented a meta-analysis to evaluate clomifene citrate (CC), metformin, and their combination's influence on anovulatory infertility treatment in PCOS patients. The aim was to define the best first-line therapy approach. 4 randomised controlled trials were included. There was no observation of fertility improvement comparing $\mathrm{CC}$ with metformin $(\mathrm{OR}=1 \cdot 22,95 \%$ CI $0 \cdot 23-6 \cdot 55, P=0 \cdot 815)$. However, statistically significant heterogeneity was noticed. There was a significant difference in fertility improvement in metformin monotherapy $(\mathrm{OR}=0 \cdot 23,95 \%$ CI $0 \cdot 14-0 \cdot 37, P<0 \cdot 0001)$ in comparison to treatment with the combination of $\mathrm{CC}$ and metformin $(\mathrm{O} R=0.99,95 \% \mathrm{CI} 0 \cdot 70-1 \cdot 40$, $P=0.982$ ). In conclusion, the combination of CC and metformin in the first-line treatment of infertility is not better than any of these medicaments in monotherapy. However, there are no specific recommendations about choosing neither of those as a first-line treatment. [19]

In 2018 Amiri et al. presented a meta-analysis comparing the effects of OCPs on the clinical and biochemical parameters of hyperandrogenism (HA) in women with PCOS. They identified applicable clinical trials from 1987 to November 2015 using electronic databases. It was observed that the duration of 3-12 months resulted in increasing in sex hormone-binding globulin levels and decreasing in Ferriman-Gallwey score, total testosterone, free testosterone, androstenedione, and dehydroepiandrosterone sulphate levels. Long-term duration (6-12 months) contributed to a more effective improvement of hirsutism therapy than the short-term (below 6 months). The best effect on treating hirsutism was noticed with OCPs containing cyproterone acetate. The outcome of this meta-analysis is conforming with the literature.[20] 
In order to guide and manage women with PCOS properly, treatment choices should be applied carefully and in accordance with patients' values and priorities. Mentioned studies presented that as a result of plentiful medicaments and managing approaches available, side effects can be minimised. Therefore, the quality of women with PCOS life is gradually enhancing.

\section{CONCLUSION}

PCOS is a multifactorial disease, therefore it is yet a lot to analyse and examine. Establishing an appropriate and effective treatment remains a great challenge. Nevertheless, various solutions and interventions enable women to contribute to their own management. Dietary and exercise implementations can easily be implemented into daily routines and are proven to have a significant effect on patients' quality of life and response to pharmacotherapy. Recommended medicaments are being continuously evaluated in terms of new combinations and approaches. There is a great number of available options to be chosen with consideration and preference of every individual. However, there is still insufficient evidence of many exploratory methods. Numerous innovative treatment options demand more randomised controlled trials with longer duration time or larger sample sizes in order to determine their significance. Future studies need to include more patients of various ages and body compositions, as the disease affects each woman differently.

\section{REFERENCES}

1. Pena, A.S., et al., Adolescent polycystic ovary syndrome according to the international evidence-based guideline. BMC Med, 2020. 18(1): p. 72.

2. Stankiewicz, M. and R. Norman, Diagnosis and management of polycystic ovary syndrome: a practical guide. Drugs, 2006. 66(7): p. 903-12.

3. Norman, R.J. and H.J. Teede, A new evidence-based guideline for assessment and management of polycystic ovary syndrome. Med J Aust, 2018. 209(7): p. 299-300.

4. Duijkers, I.J. and C. Klipping, Polycystic ovaries, as defined by the 2003 Rotterdam consensus criteria, are found to be very common in young healthy women. Gynecol Endocrinol, 2010. 26(3): p. 152-60. 
5. Pehlivanov, B. and M. Orbetzova, Characteristics of different phenotypes of polycystic ovary syndrome in a Bulgarian population. Gynecol Endocrinol, 2007. 23(10): p. 6049.

6. Timpatanapong, P. and A. Rojanasakul, Hormonal profiles and prevalence of polycystic ovary syndrome in women with acne. J Dermatol, 1997. 24(4): p. 223-9.

7. Morgante, G., et al., Polycystic ovary syndrome (PCOS) and hyperandrogenism: the role of a new natural association. Minerva Ginecol, 2015. 67(5): p. 457-63.

8. $\quad \mathrm{Wu}, \mathrm{Z} . \mathrm{H}$. , et al., Prostatic-specific antigen (PSA) levels in patients with polycystic ovary syndrome (PCOS): a meta-analysis. J Ovarian Res, 2019. 12(1): p. 94.

9. Greenwood, E.A., et al., Insulin resistance is associated with depression risk in polycystic ovary syndrome. Fertil Steril, 2018. 110(1): p. 27-34.

10. Gibson-Helm, M., et al., Delayed Diagnosis and a Lack of Information Associated With Dissatisfaction in Women With Polycystic Ovary Syndrome. J Clin Endocrinol Metab, 2017. 102(2): p. 604-612.

11. Goodman, N.F., et al., American Association of Clinical Endocrinologists, American College of Endocrinology, and Androgen Excess and Pcos Society Disease State Clinical Review: Guide to the Best Practices in the Evaluation and Treatment of Polycystic Ovary Syndrome--Part 1. Endocr Pract, 2015. 21(11): p. 1291-300.

12. Moran, L.J., et al., Lifestyle changes in women with polycystic ovary syndrome. Cochrane Database Syst Rev, 2011(7): p. CD007506.

13. Benham, J.L., et al., Role of exercise training in polycystic ovary syndrome: $a$ systematic review and meta-analysis. Clin Obes, 2018. 8(4): p. 275-284.

14. Dos Santos, I.K., et al., The effect of exercise as an intervention for women with polycystic ovary syndrome: A systematic review and meta-analysis. Medicine (Baltimore), 2020. 99(16): p. e19644.

15. Shang, Y., et al., Effect of Diet on Insulin Resistance in Polycystic Ovary Syndrome. J Clin Endocrinol Metab, 2020. 105(10).

16. Legro, R.S., et al., Diagnosis and treatment of polycystic ovary syndrome: an Endocrine Society clinical practice guideline. J Clin Endocrinol Metab, 2013. 98(12): p. 4565-92.

17. Al Khalifah, R.A., et al., Metformin or Oral Contraceptives for Adolescents With Polycystic Ovarian Syndrome: A Meta-analysis. Pediatrics, 2016. 137(5). 
18. Wu, Y., et al., Association of Metformin With Pregnancy Outcomes in Women With Polycystic Ovarian Syndrome Undergoing In Vitro Fertilization: A Systematic Review and Meta-analysis. JAMA Netw Open, 2020. 3(8): p. e2011995.

19. Palomba, S., et al., Clomiphene citrate, metformin or both as first-step approach in treating anovulatory infertility in patients with polycystic ovary syndrome (PCOS): a systematic review of head-to-head randomized controlled studies and meta-analysis. Clin Endocrinol (Oxf), 2009. 70(2): p. 311-21.

20. Amiri, M., et al., Effects of combined oral contraceptives on the clinical and biochemical parameters of hyperandrogenism in patients with polycystic ovary syndrome: a systematic review and meta-analysis. Eur J Contracept Reprod Health Care, 2018. 23(1): p. 64-77. 